\title{
Simple Technique for Repair of Avulsed Distal Biceps Tendon through One Approach. Case Series and Literature Review
}

\author{
Ahmed Refaat Khamis* \\ Consultant Orthopedics, Department of Orthopedics and Trauma Surgery, Benha \\ University Hospital, Egypt \\ *Corresponding Author: Ahmed Refaat Khamis, Consultant Orthopedics, \\ Department of Orthopedics and Trauma Surgery, Benha University Hospital, Egypt.
}

Received: September 26, 2020

Published: October 16, 2020

(C) All rights are reserved by Ahmed Refaat

Khamis.

\begin{abstract}
Introduction: Acute disruption of the distal biceps tendon is a rare injury that occurs due to eccentric contraction force on the elbow. Several techniques for reattachment of the avulsed tendon were described in the literature but no one had proved utmost efficiency over the other techniques. The purpose of this study is to evaluate the results of a simple technique for reattaching the tendon.

Methods: This is a retrospective evaluation of the use of a combined simple two fixation methods through a single anterior approach in 9 patients aiming to avoid the possible difficulties and complications reported with the other techniques.

Results: Were evaluated with the Mayo elbow score and showed 6 excellent cases (66.6\%), 2 good (22.2\%) and one fair (11.1\%). Good and comparable results to the other fixation techniques were also obtained with minimal complications.

Conclusion: The described simple combined fixation of avulsed distal biceps tendon by two anchors and trans-osseous tunnel through one anterior approach is an easy reproducible technique with minimal complications.
\end{abstract}

Keywords: Avulsion; Distal Biceps; Suture Anchors; Trans-osseous Suture

\section{Introduction}

Avulsion of the distal biceps tendon is a rare injury $(1.2: 100,000$ persons/year) occurs most commonly in the dominant elbow of men in their forties due to acute unexpected eccentric extension force on a partially flexed elbow. Smoking, diabetes, steroids, statins, and tendon impingement in radio-ulnar space with forearm rotation increase the risk of tendon degeneration and rupture [1]. The middle hypovascular area of the tendon is more subjected to rupture. The tendon is avulsed from the radial tuberosity but the bicipital aponeurosis may remain intact [1-3]. Average tendon length is $57 \mathrm{~mm}$, width is $3.6-9.0 \mathrm{~mm}$ (at level of aponeurosis). It rotates externally 90 degrees as it approaches its insertion so, the long head inserts more proximally and the short head inserts more distally. The bicipital aponeurosis extends from the tendon to the deep fascia covering the forearm flexor mass and inserts in the ulna. It directs the biceps tendon pull towards the radius while carrying the flexion force to the ulna [3]. Bicipital tuberosity of the radius is 13.8 - $30 \mathrm{~mm}$ length, 3.6 - $19 \mathrm{~mm}$ width (total area of $108 \mathrm{~mm}$ ) and sited $23 \mathrm{~mm}$ distal to the articular margin of radial head. The tendon inserts 24 degrees ulnar to the apex of radial tuberosity and incorporating it as a cam, making a pulley like action to increase the mechanical advantage of the tendon. The short head footprint is larger and the long head is more posterior so, the short head tendon is a stronger flexor-supinator with forearm in the neutral-pronated position, while the long head tendon is more supinator when the forearm is supinated 60 degrees [4]. The lateral antebrachial cutaneous nerve (LABCN) runs near the lateral aspect of the tendon. The posterior interosseous nerve (PIN) runs $10 \mathrm{~mm}$ proximal to the radial tuberosity in supination and $5 \mathrm{~mm}$ distal to it in pronation [5]. The patient usually describes an audible "pop" in the elbow with injury followed by pain and later on, ecchymosis and bulging of the biceps in the middle arm. Weakness of elbow 
supination is better assessed with the elbow flexed 90 degrees and the forearm maximally pronated to abolish the supinator muscles. The "Hook test" with the elbow flexed 90 degrees and the forearm maximally supinated, has high sensitivity and specificity. X-ray images may show an avulsed piece of bone from the radial tuberosity. MRI differentiates total from partial avulsion of the tendon [6]. Non-operative treatment is reserved for elderly patients with low physical demands and injury of the non-dominant elbow. There will be $40 \%-50 \%$ reduction in supination strength, $30 \%$ reduction in flexion strength and 15\% reduction in grip strength [6]. Surgical repair has superior regarding elbow motions strength and overall limb function [3,7-17]. Various fixation methods were described through either anterior single approach or dual anterior and posterior approaches. The fixation methods are trans-osseous suture [7], suture anchors [9,10], single far-cortical button fixation [7,11-13], double intramedullary cortical button [14] interference screws [15], screws with a cortical button [16], and endoscopically assisted repair $[10,17]$.

\section{Materials and Methods}

Through the period from June 2010 till December 2018, 9 patients with acute avulsed distal biceps tendon were treated within two weeks after injury with combined fixation by 2 suture anchors and trans-osseous suture through anterior approach. All the patients were males aged 45 - 57 years (average 51 years) and were not a heavy laborer workers. Two patient were diabetic while two patients were taking treatment for blood pressure control, and 5 patients were smokers. All the injuries were on the dominant side, 8 were right and one was left. All the cases were operated within two weeks after injury (average 9 days) because 5 patients were not presented immediately after the injury. All patients were diagnosed clinically with the hook test and confirmed by X-ray images and MRI scan of the elbow. The follow up period ranged from 12 to 36 months (average 24 months). During which the patients were assessed regarding pain and overall elbow function. X-ray images were taken after 4 weeks to assure no pull out of the anchors and the integrity of the bone at the site of tendon attachment. The final outcome was assessed by Mayo elbow performance score that involved 4 sections: pain intensity, motion, stability and function. A score of more than 9 points is excellent, 75 - 89 points is good, 60 - 74 points is fair, and less than 60 is poor [18] (Table 1). Surgical technique: Under general anesthesia with a tourniquet placed on the upper arm, the patient was placed supine and the arm on a side table with the forearm in full supination. A vertical incision about 5 $\mathrm{cm}$ length was centered over the area of the radial tuberosity starting just distal to the elbow flexion crease and should not cross it vertically. Careful subcutaneous dissection was done to find and gently retract the LABCN laterally. Deep dissection was done in the interval between the bracioradialis and the mobile wad laterally, and the common flexor-pronator mass medially. Through this interval the biceps tendon should descend to its insertion. The brachioradialis mass is retracted laterally, the flexor-pronator mass is retracted medially together with the brachial artery. Full supination of the forearm was done to identify the proximal radius and its tuberosity. No bone levers were used for retraction. Only a blunt self-retaining and Langenbeck retractors were used. Sub- periosteal dissection of the proximal radius was done to expose the foot-print of the tendon known from the remains of the fibers still attached to the bone. Two anchors $3.5 \mathrm{~mm}$ were inserted into the radial tuberosity, one at the apex of the tuberosity (short head insertion) and the other is inserted $10 \mathrm{~mm}$ proximal and just posterior to the first anchor (long head insertion). The radius must be kept fully supinated to place the anchors perpendicular to the radial tuberosity. The cortex of the area of bone between the two anchors and surrounding them should be roughened with a burr or a $2.5 \mathrm{~mm}$ drill pit to fasten the tendon-bone attachment. Still with the radius fully supinated, a 2.5 drill hole is made through the thickness of the radius on the lateral side of the middle of the tuberosity and through both cortices with care not to violate the lateral cortex. This drill hole will be vertical from anterior to posterior with the radius fully supinated and will be horizontal from medial to lateral with the radius fully pronated (Figure 1). The posterior interosseous nerve was protected with the full supination position which mobilized the nerve more posteriorly and medially away from the site of the drill hole. Thorough wound irrigation and suction is done after drilling to remove any bone debris. The avulsed tendon proximal end was almost retracted and reflected forming a loop under the biceps muscle mass in all cases. The tendon was delivered out by blunt finger insertion between the biceps and the brachialis to milk it out by unfolding the loop. This was easily done without extending the incision proximally or crossing the elbow crease in all patients except one patient whose muscle mass was big. In this patient the incision followed the elbow crease and extended for $4 \mathrm{~cm}$ proximally (Figure 2 ). The tendon end was minimally debrided from any ragged fibers or inverted tendon sheath. The sutures of the anchors was used on a separate needle to make 5 anchoring sutures with each thread in the tendon. The sutures were tightened and the tendon was pulled down to the anchors sites. A separate suture (No 1 Ethibond) was used to secure 
the tendon through the preformed drill hole (Figure 3). The field was further copiously irrigated and the repair was tested by rotation of the forearm and flexion-extension of the elbow. Few more stitches were taken between the tendon and any remaining tissue at its footprint. The final construct resembled a triangular 3 points of fixation of the tendon with the 2 anchors forming the base of the triangle. The fixation point through the drill hole will secure the fixation more and counteracts the pulling stresses on the anchors by the tendon with elbow motions. The subcutaneous tissue and the skin were closed without drain. An above elbow back slab in 90 degrees flexion was applied for 2 weeks till the skin stitches were removed then a complete above elbow cast was applied for 2 more weeks. Rehabilitation protocol started after 4 weeks with gradual flexion-extension and pronation-supination active exercises without weights for 2 weeks followed by the same exercises with light weights $(0.9 \mathrm{Kg})$ for 4 weeks (Figure 4$)$.

\begin{tabular}{|l|c|c|}
\hline Function & Points & Definition (points) \\
\hline Pain & 45 & $\begin{array}{c}\text { None (45) } \\
\text { Mild (30) } \\
\text { Severe (0) }\end{array}$ \\
\hline Motion & 20 & $\begin{array}{c}\text { Arc }>100 \text { degrees (20) } \\
\text { Arc 50-100 degrees (15) } \\
\text { Arc < 50 degrees (5) }\end{array}$ \\
\hline Stability & 10 & $\begin{array}{c}\text { Stable (10) } \\
\text { Moderate instability (5) } \\
\text { Gross instability (0) }\end{array}$ \\
\hline Function & 25 & $\begin{array}{c}\text { Comb hair (5) } \\
\text { Feed (5) }\end{array}$ \\
& & $\begin{array}{c}\text { Perform hygiene (5) } \\
\text { Don shirt (5) }\end{array}$ \\
\hline Total & 100 & Don shoe (5) \\
\hline
\end{tabular}

Table 1: Mayo elbow performance score: excellent $>90$, good 57 89 , fair $60-74$, poor $<60$.

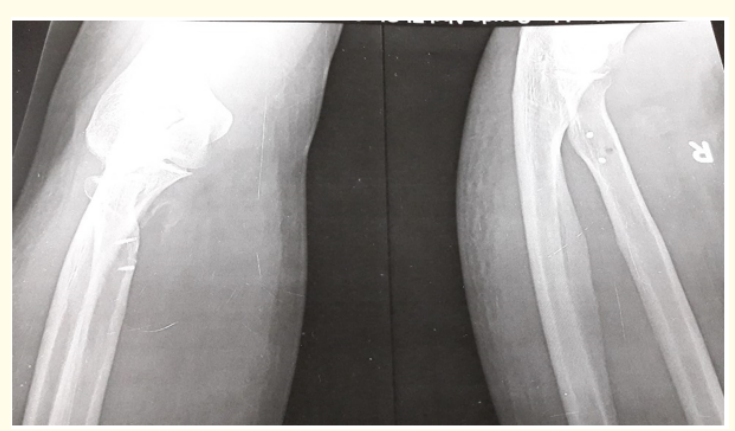

Figure 1: X-ray image showing position of the anchors and the drill hole.

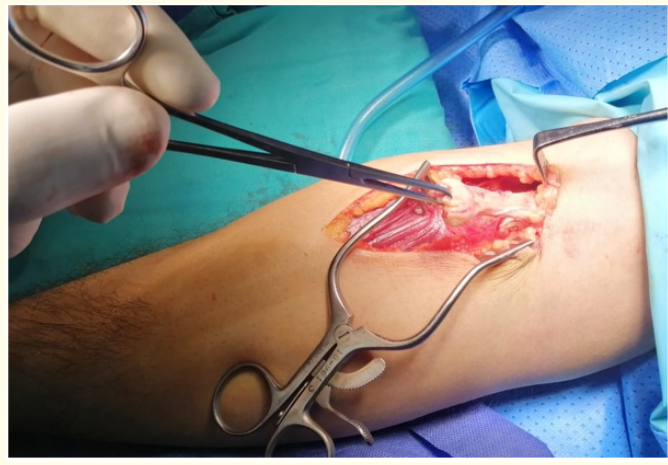

Figure 2: Intraoperative photo with tendon delivered out.

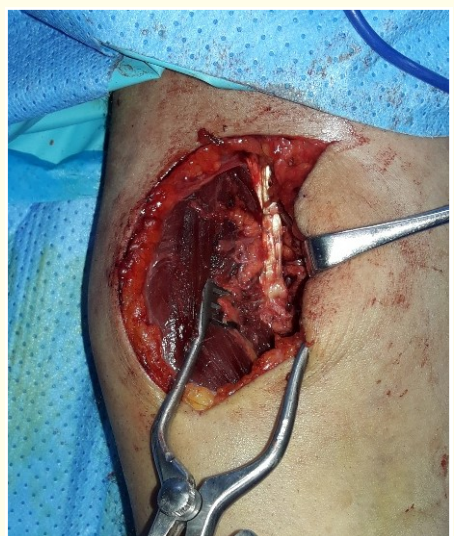

Figure 3: Intraoperative photo of the tendon after re-attachment.
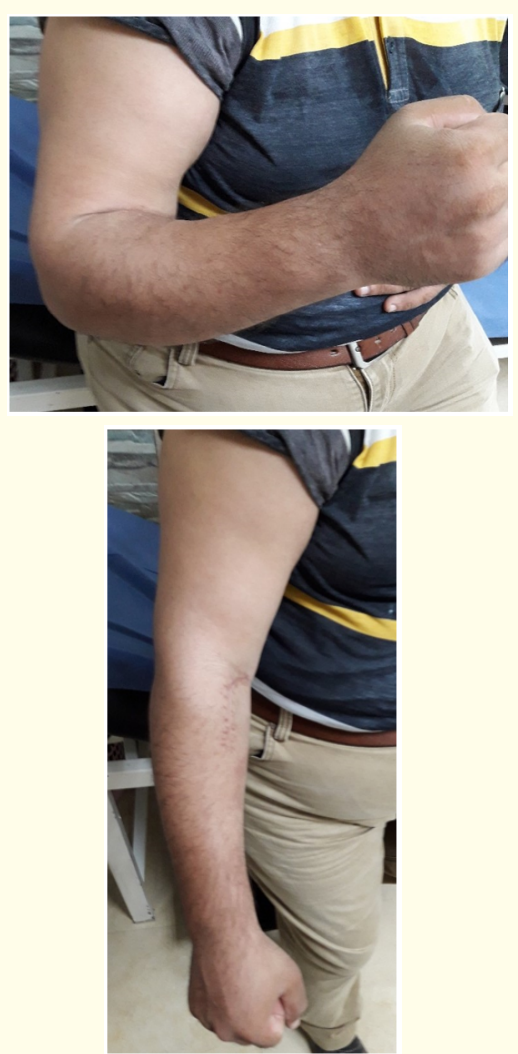

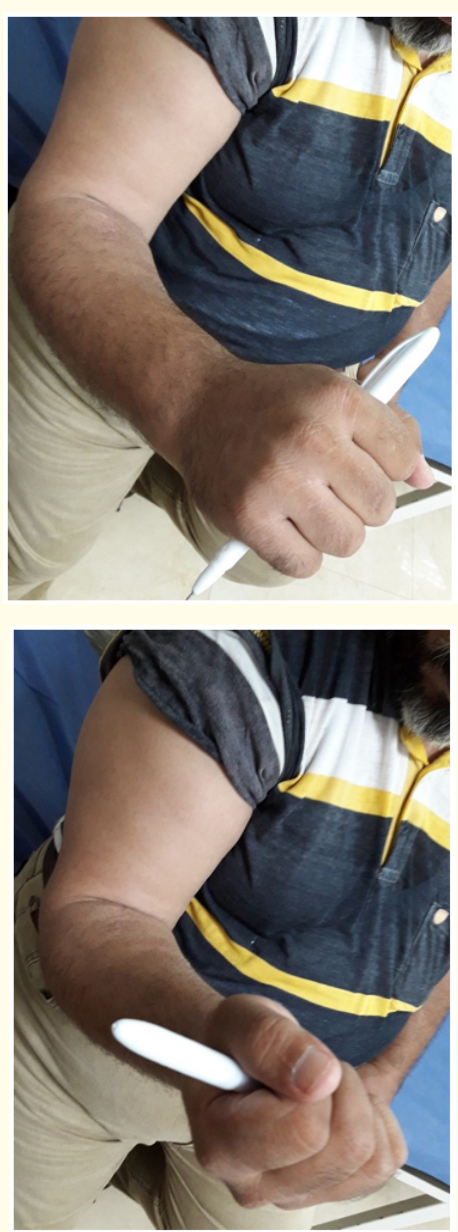

Figure 4: Active range of motion during follow up. A, Flexion. B, Extension. C, Pronation. D, Supination.

\section{Results}

The results were assessed clinically by the Mayo elbow performance score (Table 1) and radiologically by X-ray images to confirm the position of the anchors during the follow up period. The range of motion in all patients was assessed with a goniometer in comparison to the other (non-operated) elbow. All the patients had the injury on the dominant side after an attempt to lift a heavy object with the elbow in extension and supination. The patient's age ranged from 45 to 57 years (average 51 years), all had the injury on the dominant side and all were males (Table 2). The overall results of the 9 patients on the Mayo score were: 6 excellent (66.6\%), 2 good (22.2\%) and one fair (11.1\%). No patient was reported immediately to the hospital. Six patients were presented within one week after injury (66.6\%), two patients presented within 2 weeks $(22.2 \%)$, and one patient (11.1\%) was presented 15 days after injury. Two patients were diabetics (22.2\%), 5 were smokers (55.5\%) and one was dyslipidemic (11.1\%) and was taking statins. The patient with the fair result was smoker, diabetic and hypertensive. The two patients with good results were smokers and one of them was also diabetic (Table 2). The overall rate of complications in the 9 patients was 33.3\% (3 patients). Two patients had LABCN neuropathy (22.2\%), of these one was transient (11.1\%), and two patients had wound infection (22.2\%), of these one was superficial and did not need a second operation. No patients had injury of the PIN and no patients developed HO in the follow up period. One of the two patients with good results was diabetic and had superficial infection in the wound. This was managed with antibiotics and healed soundly. He had mild pain and his elbow flexion range decreased to 90 degrees. The other patient with good result had transient neuropathy of the LABCN that was resolved 6 weeks after surgery. His elbow flexion range was 100 degrees. Nevertheless, both patients had full pronation-supination range of motion. The patient with a fair result was 57 years old, diabetic, hypertensive, dyslipidaemic and smoker. He had postoperative neuropathy of the LABCN that was not resolved through the follow up period (Table 2 ). He had also wound infection that necessitated reoperation after 5 days for wound debridement and secondary sutures. This was attributed to his diabetes and smoking habit. This patient had mildto-moderate pain with elbow motions, his arc of motion was flexion till degrees, supination was reduced to 30 degrees, his elbow was stable, and his elbow function was affected to a degree reflected on his job as a barber. No tendon re-rupture occurred in the 9 patients.

\begin{tabular}{|c|c|c|c|c|c|c|c|c|c|}
\hline $\begin{array}{c}\text { Age: } \\
\text { years }\end{array}$ & $\begin{array}{c}\text { Injured } \\
\text { side }\end{array}$ & Job & $\begin{array}{c}\text { Time to } \\
\text { surgery: } \\
\text { days }\end{array}$ & Comorbidity & Smoking & $\begin{array}{c}\text { Follow up } \\
\text { period: } \\
\text { months }\end{array}$ & $\begin{array}{c}\text { Final Out- } \\
\text { come }\end{array}$ & $\begin{array}{c}\text { Mayo } \\
\text { score }\end{array}$ & Complications \\
\hline 50 & Lt & Engineer & 11 & Diabetes & + & 12 & Good & 85 & Superficial infection \\
\hline 45 & Rt & Employee & 2 & - & - & 18 & Excellent & 100 & - \\
\hline 54 & Rt & Employee & 4 & Hypertensive & + & 12 & Excellent & 94 & - \\
\hline 46 & Rt & Employee & 5 & - & - & 12 & Excellent & 97 & - \\
\hline 49 & Rt & Employee & 7 & - & - & 30 & Excellent & 95 & - \\
\hline 53 & Rt & Employee & 7 & - & + & 12 & Excellent & 97 & - \\
\hline 53 & Rt & Employee & 10 & Hypertensive & + & 12 & good & 80 & $\begin{array}{c}\text { LABCN neuropathy; } \\
\text { transient }\end{array}$ \\
\hline 52 & Rt & Employee & 5 & - & - & 24 & Excellent & 95 & - \\
\hline 57 & $\mathrm{Rt}$ & Barber & 15 & Diabetes & + & 36 & Fair & 74 & $\begin{array}{c}\text { LABCN neuropathy; } \\
\text { permanent. }\end{array}$ \\
& & & & Hypertension & & & & & $\begin{array}{c}\text { Wound infection re- } \\
\text { quired reoperation. }\end{array}$ \\
\hline
\end{tabular}

Table 2: Results. 


\section{Discussion}

Controversy exists clearly in the literature about surgical management of completely avulsed distal biceps tendon regarding the best fixation technique nd surgical approach. Systematic reviews were comparing the different fixation methods and the two approaches (anterior or dual incision). Watson., et al. [19] reported complications rate $26.4 \%$ for suture anchors, $20.4 \%$ for bone tunnels, $44.8 \%$ for intraosseous screws, and $0 \%$ for cortical button fixation. Cortical button fixation was used in only $3.5 \%$ of patients. This small sample size is inadequate to compare complication rates with the other techniques. They also found that the two-incision approach has less complications but the number of patients was small, made the studies underpowered to compare the two approaches. Kodde., et al. [20] reported no significant difference in strength or range of motion between the 4 fixation methods. Also, the double incision approach showed significantly less complications, and the trans-osseous tunnel fixation had much less complications than the other techniques. El-Hawary., et al. [21] reported significantly more elbow flexion with the one incision approach with significant increase in the isokinetic and isometric flexion strength in early follow-up compared with the dual approach. However, the two groups became equalized after one year. Chavan., et al. [22] reported no significant difference in the rate of complications between the two approaches, but more loss of forearm rotation was reported with the dual approach. Johnson., et al. [23] reported no statistically significant differences in flexion strength, supination strength, or complications between single incision with suture anchors fixation and 2 incisions with bone tunnel fixation. Reattachment of avulsed distal biceps tendon by a cortical button was first described by Bain., et al. [11]. Higher load to failure of the cortical button fixation was proved by anatomical and biomechanical studies, allowing for immediate unrestrictive postoperative active motion [18-20,24,25] and gave excellent clinical-functional outcome with less complications $[10,11,21,22]$. Studies reported on complications and outcomes of endobutton fixation included small numbers of patients with low levels of evidence despite the reported good results [7,26-31]. Several complications were reported such as heterotopic ossification (HO), PIN injury, LABCN injury, and failure of repair [25,32-36]. PIN palsy is a serious, relatively rare, complication of cortical button fixation [35,36]. Wrong guide pin placement [37,38], PIN incarceration by the button [39], or nerve compression by bone levers placed on either sides of the proximal radius are the possible etiologies. Nigro., et al. [36] reported a $5 \%$ incidence of PIN injury in 9 of 180 patients, 6 of them were fixed by endobutton through the anterior approach $3.3 \%$ PIN injury). Late reconstruction of the tendon is associated with more complications. Cain., et al. [28] reported $6 \%$ of PIN injury, $30 \%$ of LABCN injury and $3 \%$ of superficial radial nerve (SRN) injury in 198 patients (119 acute and 79 chronic ruptures) fixed with three different methods (anchors, bone tunnels, and endobutton) through an anterior approach in $93 \%$ of them. Most cases of LABCN palsy resolved spontaneously. Smith and Amirfeyz [40] reported transient paresthesia in two-thirds of their patients $(27 \%$ of SRN and $36 \%$ of LABCN). They explained this by the sensitivity of their measurement technique by a 0 -to-10 analog scale compared with the un-operated arm to the degree that it can detect mild sensory deficits that the patient is unaware by it. Heterotopic ossification may occur after repair due to bone debris of drilling, hematoma formation, and initial muscle injury or after surgical dissection. The risk decreases with single-incision approach, minimal muscle dissection, minimal bone drilling and copious irrigation of the surgical field [32,34,41]. Agrawal and Stinson [32] excised the exostosis 5 months postoperatively due to 20-degrees loss in supination. Followed by indomethacin for 6 weeks. Dillon and Lepore [34] used conservative treatment and reported satisfactory outcome after one year. Vidal., et al. [41] reported 50\% incidence of HO in 4 of 8 patients fixed with cortical button through single incision and excised the HO in three patients. Cain., et al. [28] reported a 3\% incidence of HO with cortical button fixation and the same percentage with sutures anchors. They also reported pull out of the screw and fracture of radius with the transfixion screw technique. Kodde., et al. [20] reported 13\% HO with cortical button fixation, but it was severe only in $1.3 \%$ Re-rupture after tendon repair is uncommon $[6,22,26]$. Cain., et al. [28] reported no re-ruptures with endo-button repairs and 3.3\% re-ruptures in 119 patients with suture anchor fixation. Kodde., et al. [31] reported 1.3\% re-ruptures in 146 endo-button repairs. Peeters., et al. [42] concluded excellent outcome with fixation by endobutton however, they reported disengagement of the endobutton in 3 patients, and they had to remove the endobutton in another patient. Banerjee., et al. [26] reported an unusual high rate of complications (59\%) after cortical button biceps repair only in acute cases (27 patients) including PIN palsy, LABCN palsy that did not recover, re-rupture, HO, wound healing problems and revision surgery. They attributed this to the use of Hohmann retractors on both sides of the radius, the trajectory of the drilling (more radially and distally), the length of the skin incision, and different operating surgeons, both experienced and not. A more vertical pin trajectory with the elbow in extension and full 
supination, smaller incision, use of skin hooks instead of retractors, intraoperative fluoroscopy for correct button placement and thorough irrigation of the wound after drilling are the recommended measures to prevent those complications. Prophylactic radiation and/or indomethacin administration may be recommended to avoid heterotobic ossification $[6,22,26,28]$. In the current study there were LABCN palsy in $22.2 \%$ of patients ( 2 cases, one of them resolved spontaneously), no PIN or SRN palsy and no heterotopic ossification. However, 2 cases had infection (22.2\%) one was superficial and the other was deep and needed reoperation for wound debridement. The rate of complications in this study is near the rates reported by Watson., et al. Chavan., et al. and Johnson., et al. but less than the rate reported by Nigro., et al. and Smith and Amirfeyz. Recordon., et al. [8] in a retrospective comparative study between cortical button (19 patients) and transosseous suture fixation (27 patients) through a 2 -incision approach reported no significant statistical differences in subjective patient evaluation, pain, range of motion, supination strength, and overall complications. Despite immobilization in a cast for 6 weeks in the trans-osseous group, the clinical outcome they reported was similar at the latest follow-up. In the current study, all patients were immobilized for 4 weeks before starting gradual rehabilitation. The period of immobilization had no negative impact on the final outcome. This is corresponding to the recommendations by Recordon., et al. [8]. However, there is no consensus in literature on the postoperative rehabilitation protocol and some surgeons prefer application of long arm cast or splint in 90 degrees flexion for 1 - 2 weeks followed by active range of motion (ROM), and heavy lifting is allowed after 2 - 3 months. Two studies limited extension to 30 - 40 degrees for 3 - 6 weeks. Other surgeons prefer the use of a long arm cast for 6 weeks despite the proven strength of endo-button fixation. Spencer Jr., et al. [43] reported a longer time to achieve full ROM with the supervised physiotherapy compared to unrestricted ROM protocol (8.6 and 4.3 weeks respectively). This suggests that unrestricted ROM allows faster return to full ROM after fixation with cortical button [40]. Panagopoulos., et al. [44] studied the outcome and complications of only cortical button fixation of distal biceps tendon in a systematic review. They reported satisfactory functional ROM in about $85 \%$ of patients. The most common complication was transient nerve palsy and can be avoided by appropriate surgical technique. However, they noted that the clinical superiority of cortical button fixation in comparison to other techniques has not yet confirmed in the literature. Huynh., et al. [46] recently reviewed the results of 60 cases of distal biceps tendon avulsion repaired with the cortical button technique. They found relatively high rate of complications (5\% re-rupture, 56.7\% HO mostly Brooker class-I, and 11.7\% LABCN paresthesia). In their cases there was associated minimal loss of elbow flexion and supination ( $96 \%$ and $91 \%$ of those on contralateral side) together with 5 degrees loss of pronation. These results caused a minimal degree of disability as reflected by the DASH scores. Camp., et al. used a single intramedullary cortical button to fix the distal biceps tendon through one small drill hole [45]. They concluded that this technique avoids the complications and the risk of fracture of the proximal radius with the far cortical button tenodesis. In this case series, the combined re-attachment of the distal biceps tendon with two suture anchors and one trans-osseous suture was able to avoid most of the reported complications of using each technique separately. Also, it combined the biomechanical and surgical advantages of both techniques through a less invasive single anterior approach. By this combined technique, it was possible to avoid the reported drawbacks of re-attaching the tendon by the cortical endo-button technique in which, the $6 \mathrm{~mm}$ drill hole for the passage of the tendon inside the bone will weaken this small part of the radius and may predispose to fracture. A very precise trajectory of drilling is required to avoid injury to the PIN. Also, the blind flipping of the endobutton on the posterior cortex of the radius may press on the PIN. More drilling of the bone is required for a cortical button fixation in comparison to fixation by a transosseous suture. This may increase the risk of HO. The single incision technique avoids the possible complication of HO happens with the 2 incisions technique. Many orthopedic surgeons has limited experience in reattachment of the avulsed distal biceps tendon due to the rarity of the injury. The need for a simple technique of repair without the risk of potential complications and that does not need deep experience by the surgeon is required. The current technique is easy to perform and allows postoperative rehabilitation without the fear of weakening of the proximal radius and fracturing it especially in the obese or heavy muscular patients.

\section{Weakness points of this study}

The number of patients is small. This is attributed to the rarity of the injury. Most of the reported case series of repair of distal biceps tendon avulsion in the literature involved small number of cases. Further studies with larger number of patients using the same technique are required. Also, abiomechanical study of the ultimate strength of this form of combined fixation is required. 


\section{Conclusion}

Controversy still exists about the proper technique of fixation of the avulsed distal biceps tendon. All the techniques described in literature has some complications. The cortical button fixation technique through anterior approach had been believed to be without complications in some studies and allows immediate postoperative motion however, recent reviews of the results of this technique showed some serious complications. Whatever the technique of fixation is, the target of treatment is to restore as much as possible of the normal power and range of motion in comparison to the un-injured side. The current technique is a combination of two already existed fixation methods in order to improve the tendon to bone attachment at its anatomical foot print to prevent re-rupture. This combined fixation method avoids the complications of PIN injury and HO. The anterior approach protects against radioulnar synostosis as it avoids dissection through the interosseous membrane however, it may be associated with temporary LABC nerve paresthesia.

\section{Bibliography}

1. MR Safran and SM Graham. "Distal biceps tendon ruptures: incidence, demographics, and the effect of smoking". Clinical Orthopaedics and Related Research 404 (2002): 275-283.

2. C Savvidou and R Moreno. "Spontaneous distal biceps tendon ruptures: are they related to statin administration?" Hand Surgery 17.2 (2012): 167-171.

3. Cucca YY., et al. "The biceps brachii muscle and its distal insertion: observations of surgical and evolutionary relevance". Surgical and Radiologic Anatomy 32.4 (2010): 371-375.

4. van den Bekerom J., et al. "Clinical relevance of distal biceps insertional and footprint anatomy". Knee Surgery, Sports Traumatology, Arthroscopy 24.7 (2016): 2300-2307.

5. Hackl M., et al. "The course of the posterior interosseous nerve in relation to the proximal radius: is there a reliable landmark?" Injury 46.4 (2015): 687-692.

6. DD Savin., et al. "Surgical Management of Acute Distal Biceps Tendon Ruptures, Current concepts review". Journal of Bone and Joint Surgery 99 (2017): 785-796 d.

7. M Citak., et al. "Surgical repair of the distal biceps brachii tendon: a comparative study of three surgical fixation techniques". Knee Surgery, Sports Traumatology, Arthroscopy 19.11 (2011): 1936-1941.
8. JA Recordon., et al. "Endobutton versus transosseous suture repair of distal biceps rupture using the two-incision technique: a comparison series". Journal of Shoulder and Elbow Surgery 24.6 (2015): 928- 933.

9. L Balabaud., et al. "Repair of distal biceps tendon ruptures using a suture anchor and an anterior approach". The Journal of Hand Surgery British 29.2 (2004): 178-182.

10. T Gregory., et al. "Repair of distal biceps tendon rupture using a suture anchor: description of a new endoscopic procedure". The American Journal of Sports Medicine 37.3 (2009): 506-511.

11. GI Bain., et al. "Repair of distal biceps tendon rupture: a new technique using the Endobutton". Journal of Shoulder and Elbow Surgery 9.2 (2000): 120-126.

12. JA Greenberg., et al. "EndoButton-assisted repair of distal biceps tendon ruptures". Journal of Shoulder and Elbow Surgery 12. 5 (2003): 484- 490.

13. J King and M Bollier. "Repair of distal biceps tendon ruptures using the EndoButton". Journal of the American Academy of Orthopaedic Surgeons 16.8 (2008): 490-494.

14. S Siebenlist., et al. "The double intramedullary cortical button fixation for distal biceps tendon repair". Knee Surgery, Sports Traumatology, Arthroscopy 19.11 (2011): 1925-1929.

15. W Khan., et al. "Repair of distal biceps tendon rupture with the Biotenodesis screw". Archives of Orthopaedic and Trauma Surgery 124.3 (2004): 206-208.

16. AD Heinzelmann., et al. "A combined technique for distal biceps repair using a soft tissue button and biotenodesis interference screw". The American Journal of Sports Medicine 37.5 (2009): 989-994.

17. S Sharma and G MacKay. "Endoscopic repair of distal biceps tendon using an EndoButton". Arthroscopy 21.7 (2005): 897. e1-897.e4.

18. Cusick MC., et al. "Accuracy and reliability of the Mayo Elbow Performance Score”. Journal of Hand Surgery 39.6 (2014): 1146-1150.

19. JN Watson., et al. "Repair techniques for acute distal biceps tendon ruptures: a systematic review". The Journal of Bone and Joint Surgery-American Volume 96.24 (2014): 2086-2090.

20. IF Kodde., et al. "Re-fixation techniques and approaches for distal biceps tendon ruptures: a systematic review of clinical studies". Journal of Shoulder and Elbow Surgery 25.2 (2016): e29-e37. 
21. R El-Hawary., et al. "Distal biceps tendon repair: comparison of surgical techniques". The Journal of Hand Surgery 28.3 (2003): 496-502.

22. PR Chavan., et al. "Repair of the ruptured distal biceps tendon: a systematic review". American Journal of Sports Medicine 36.8 (2008): 1618-1624.

23. TS Johnson., et al. "One-versus two-incision technique for distal biceps tendon repair". HSS Journal 4.2 (2008): 117-122.

24. PM Sethi and JE Tibone. "Distal biceps repair using cortical button fixation". Sports Medicine and Arthroscopy Review 16.3 (2008): 130-135.

25. DM Rose., et al. "Biomechanical analysis suggests early rehabilitation is possible after single-incision EndoButton distal biceps repair with FiberWire". Knee Surgery, Sports Traumatology, Arthroscopy 19.6 (2011): 1019-1022.

26. M Banerjee., et al. "High complication rate following distal biceps refixation with cortical button". Archives of Orthopaedic and Trauma Surgery 133.10 (2013): 1361-1366.

27. HA Bosman., et al. "Anatomic direct repair of chronic distal biceps brachii tendon rupture without interposition graft". Journal of Shoulder and Elbow Surgery 21.10 (2012): 1342-1347.

28. RA Cain., et al. "Complications following distal biceps repair". The Journal of Hand Surgery 37.10 (2012): 2112-2117.

29. MT Dillon., et al. "Repair of acute and chronic distal biceps tendon ruptures using the EndoButton". Hand 6.1 (2011): 39-46.

30. R K Gupta., et al. "Repair of the torn distal biceps tendon by endobutton fixation". Indian Journal of Orthopaedics 46.1 (2012): 71-76.

31. IF Kodde., et al. "Reconstruction of distal biceps tendon ruptures with a cortical button". Knee Surgery, Sports Traumatology, Arthroscopy 23.3 (2015): 919-925.

32. V Agrawal and M J Stinson. "Case report: Heterotopic ossification after repair of distal biceps tendon rupture utilizing a single-incision Endobutton technique". Journal of Shoulder and Elbow Surgery 14.1 (2005): 107-109.

33. SS Desai., et al. "Failed distal biceps tendon repair using a single-incision EndoButton technique and its successful treatment: case report". Journal of Hand Surgery American 35.12 (2010): 1986-1989.
34. MT Dillon and DJ Lepore. "Heterotopic ossification after singleincision distal biceps tendon repair with an endobutton". Journal of Surgical Orthopaedic Advances 20.3 (2011): 198-201.

35. MR Fajardo., et al. "Multiple nerve injuries following repair of a distal biceps tendon rupture: case report and review of the literature". Bulletin of the Hospital for Joint Diseases 71.2 (2013): 166-169.

36. PT Nigro., et al. "Prognosis for recovery of posterior interosseous nerve palsy after distal biceps repair". Journal of Shoulder and Elbow Surgery 22.1 (2013): 70-73.

37. EY Lo., et al. "The effect of drill trajectory on proximity to the posterior interosseous nerve during cortical button distal biceps repair". Arthroscopy 27.8 (2011): 1048-1054.

38. N Thumm., et al. "Proximity of the posterior interosseous nerve during cortical button guidewire placement for distal biceps tendon reattachment". The Journal of Hand SurgeryAmerican 40.3 (2015): 534-536.

39. J van den Bogaerde and E Shin. "Posterior interosseous nerve incarceration with endobutton repair of distal biceps". Orthopedics 38.1 (2015): e68-e71.

40. JRA Smith and R Amirfeyz. "Does immediate elbow mobilization after distal biceps tendon repair carry the risk of wound breakdown, failure of repair, or patient dissatisfaction". Journal of Shoulder and Elbow Surgery 25.5 (2016): 810- 815.

41. AF Vidal., et al. "Extensive heterotopic ossification after suspensory cortical fixation of acute distal biceps tendon ruptures". Arthroscopy 28.7 (2012): 1036-1040.

42. T Peeters., et al. "Functional outcome after repair of distal biceps tendon ruptures using the endobutton technique". Journal of Shoulder and Elbow Surgery 18.2 (2009): 283- 287.

43. EE Spencer Jr., et al. "Is therapy necessary after distal biceps tendon repair?" Hand 3.4 (2008): 316-319.

44. A Panagopoulos., et al. "Clinical Outcomes and Complications of Cortical Button Distal Biceps Repair: A Systematic Review of the Literature". Journal of Sports Medicine (2016).

45. CL Camp., et al. "Single incision technique for repair of distal biceps tendon avulsions with intramedullary cortical button". Arthroscopy Techniques 5.2 (2016): e303-e307. 
46. T Huynh., et al. "Outcomes and Complications After repair of Complete Distal Biceps Tendon Rupture with the Cortical Button Technique". JBJS Open Access (2019): e0013.

\section{Assets from publication with us}

- Prompt Acknowledgement after receiving the article

- Thorough Double blinded peer review

- Rapid Publication

- Issue of Publication Certificate

- High visibility of your Published work

Website: https://www.actascientific.com/

Submit Article: https://www.actascientific.com/submission.php

Email us: editor@actascientific.com

Contact us: +919182824667 